\title{
O problema da elaboração de grade de horários escolares: uma aplicação à Universidade Federal do Espírito Santo
}

\author{
Rafael Marin Permanhane \\ Graduando do Curso de Bacharelado em Matemática, CEUNES, UFES, \\ 29930-000, São Mateus, ES \\ E-mail: rafael_marinp@hotmail.com, \\ Leonardo Delarmelina Secchin \\ Departamento de Matemática Aplicada, CEUNES, UFES \\ 29930-000, São Mateus, ES \\ E-mail: lsecchin@ceunes.ufes.br.
}

\section{RESUMO}

O problema da elaboração de grade de horários consiste na designação de um conjunto de disciplinas entre professores e salas de aula, durante um período de tempo pré-definido. É objeto de estudo de vários pesquisadores. Dentre eles, MirHassani [3] e Daskalaki e Birbas [2]. Ambos os trabalhos modelam o problema via programação inteira. MirHassani propõe uma formulação simplificada e eficiente do ponto de vista computacional. Já Daskalaki e Birbas concebem um modelo bem mais completo, e evidentemente, de maior complexidade computacional.

Utilizamos um modelo de programação inteira, adaptando o trabalho de Daskalaki e Birbas para a realidade do Departamento de Matemática Aplicada da Universidade Federal do Espírito Santo. A resolução do modelo é feita em duas fases: a primeira aloca disciplinas aos professores levando em consideração, dentre outras coisas, as preferências e o balanceamento de carga horária dos professores; na segunda fase os horários são estabelecidos, buscando compacidade e cuidando dos turnos de trabalho, dentre outras coisas. Cabe ressaltar que Daskalaki e Birbas não tratam questões de condensação de horários e turnos de trabalho. Dados reais do semestre letivo 2012/2 foram utilizados.

Palavras-chave: problema de horários, programação inteira, timetabling.

\section{Elementos da modelagem do problema}

No que segue, apresentamos os principais elementos da modelagem. Para o modelo da primeira fase, definimos as variáveis binárias $x_{p c}=1$ se, e somente se o professor $p$ ministra a disciplina $c$; $y_{p g}=1$ se, e somente se, o professor $p$ ministra disciplinas do grupo de disciplinas $g$; e $w_{p}=1$ se o professor $p$ ministra disciplinas no período noturno. Ainda, definimos $e q \geq 0$ como limitante inferior para carga horária dos professores que não possuem atividades de coordenação/chefia. Parâmetros $\operatorname{PREF}[p, g]$ de preferência dos professores por grupos de disciplinas são definidos, e adicionados à função objetivo, que vem dada por

$$
\min \sum_{p} \sum_{g} \sum_{c ; G[c]=g}\left(x_{p c} \cdot \operatorname{PREF}[p, g]\right)-500 \cdot e q
$$

onde $G[c]$ é o conjunto das disciplinas do grupo $g$.

Além das restrições inerentes ao problema, como aquela que evita um professor ser alocado para aulas em duas disciplinas para um mesmo horário, inserimos restrições adicionais, as quais 
relatamos algumas a seguir. O equilíbrio da carga horária de trabalho entre professores é feito com auxílio da restrição

$$
\sum_{c}\left(2,5 \cdot \mathrm{CH}[c] \cdot x_{p c}\right)+\mathrm{CHadicional}[p] \geq e q
$$

onde CHadicional $[p]$ é a carga horária de pesquisa e extensão do professor $p$, e $\mathrm{CH}[c]$ a carga horária semanal da disciplina $c$.

A fim de evitar um número excessivo de disciplinas com programas distintos alocadas a um professor, limitamos o número de grupos para cada professor fazendo $\sum_{g} y_{p g} \leq 2$. Assim, disciplinas de um mesmo grupo devem ser entendidas como aquelas de programas similares.

Outras restrições impedem que um professor ministre aulas simultaneamente pela manhã e noite; limita o número de disciplinas noturnas por professor; aloca obrigatoriamente grupo de disciplinas específicas para um professor especialista, quando for de decisão do Departamento; e impedem que um professor ministre duas ou mais disciplinas distintas para uma mesma turma principal (entende-se por turma principal aquela formada por alunos periodizados).

No que segue, faremos observações acerca do modelo da segunda fase. Primeiramente, esta fase depende da divisão de disciplinas entre os professores. Consideremos portanto uma solução ótima $\left(x_{p c}, y_{p g}, w_{p}, e q\right)$ da primeira fase. Definimos as novas variáveis binárias $\bar{x}_{c h d}=1$ se, somente se, a disciplina $c$ é dada no horário $h$ do dia $d ; \bar{y}_{c d}=1$ se, e somente se, a disciplina $c$ é dada no dia $d ; \bar{w}_{m d}=1$ se, e somente se, a turma $m$ tem aula no dia $d$; e $\bar{z}_{p d}=1$ se, e somente se, o professor $p$ dá aula no dia $d$. Definimos ainda as variáveis inteiras $\operatorname{hmin}_{p}$ e $\operatorname{hmax}_{p}$ que prestam a condensar os horários semanais do professor $p$ no intervalo [ $\mathrm{hmin}_{p}, \mathrm{hmax}_{p}$ ].

A função objetivo da segunda fase é dada por

$$
\min \sum_{c, d}\left[0,3\left(\bar{x}_{c 0 d}+\bar{x}_{c 15 d}\right)+0,1\left(\bar{x}_{c 1 d}+\bar{x}_{c 14 d}\right)\right]-\sum_{d, c ; \mathrm{CH}[c]=4} 10 \cdot \bar{y}_{c d}+\sum_{p}\left(\operatorname{hmax}_{p}-\operatorname{hmin}_{p}\right)
$$

A primeira soma procura evitar a escolha dos dois primeiros horários da manhã $(h \in\{0,1\})$ e dos dois últimos da noite $(h \in\{14,15\})$. Observe que os fatores que multiplicam as variáveis são positivos e pequenos. O objetivo é deslocar horários somente quando não há prejuízos à distribuição global das disciplinas. A segunda soma evita que disciplinas tenham quatro horários seguidos, e a terceira procura condensar os horários semanais de cada professor.

Nesta fase adicionamos restrições para, dentre outras coisas, limitar o número de dias da semana para uma disciplina; garantir, para cada professor, janelas de tempo vagas nos horários de almoço e jantar; proibir que uma disciplina tenha aulas fora de seu turno pré-definido; garantir que os horários de uma disciplina em um mesmo dia sejam dados consecutivamente; proibir aulas de certas disciplinas em dias consecutivos; deixar livre horários pré-fixados para reuniões; e limitar o número de dias com aulas de um professor. Em especial, as disciplinas de uma turma principal devem ser ministradas em único turno. Impomos assim que

$$
\forall m, d, \quad \bar{w}_{m d} \leq \sum_{h, c ; \mathrm{T}[c]=m} \bar{x}_{c h d} \leq N \cdot \bar{w}_{m d}
$$

onde T $[c]$ é a turma da disciplina $c$ e $N$ um inteiro suficientemente grande. Outra restrição que destacamos é quanto ao espaço físico. Limitamos as aulas em um mesmo horário pelo número $S$ de salas disponíveis

$$
\forall d, h, \quad \sum_{c} \bar{x}_{c h d} \leq S
$$

A condensação dos horários semanais de cada professor é feita pelas variáveis $\operatorname{hmax}_{p} \mathrm{e} \mathrm{hmin}_{p}$, como comentamos. Definimos essas variáveis considerando três intervalos de horários: $[0,5]$, dos primeiros horários da manhã; [6,10], do fim da manhã a meados da tarde; $[7,11]$, do início ao fim da tarde; e $[12,15]$, do fim da tarde ao fim da noite. As restrições a seguir garantem que 
a diferença $\operatorname{hmax}_{p}-\operatorname{hmin}_{p}$ seja não negativa e represente satisfatoriamente a condensação de horários dos professores:

$$
\begin{array}{cc}
\forall p ; w[p]=0, d, h ; 0 \leq h<6 & \sum_{c}\left(x_{p c} \cdot h\right) \cdot \bar{x}_{c h d} \geq \operatorname{hmin}_{p} \\
\forall p ; w[p]=1, d, h ; 6 \leq h<11 & \sum_{c}\left(x_{p c} \cdot h\right) \cdot \bar{x}_{c h d} \geq \operatorname{hmin}_{p} \\
\forall p ; w[p]=0, d, h ; 7 \leq h<12 & \sum_{c}\left(x_{p c} \cdot h\right) \cdot \bar{x}_{c h d} \leq \operatorname{hmax}_{p} \\
\forall p ; w[p]=1, d, h ; 12 \leq h<16 & \sum_{c}\left(x_{p c} \cdot h\right) \cdot \bar{x}_{c h d} \leq \operatorname{hmax}_{p}
\end{array}
$$

Lembramos que $w_{p}$ é variável da primeira fase igual a 1 se o professor $p$ ministra aulas noturnas.

\section{Resultados}

Os dados utilizados englobam 18 professores, 49 disciplinas distribuídas em 29 grupos e 36 turmas principais. Foi utilizado um computador Intel Core 2 Quad $2.33 \mathrm{GHz}, 2 \mathrm{~Gb}$ de memória RAM, sistema Linux 64 bits com o CPLEX 12.5.1. Na primeira fase, chegamos à otimalidade em 3,22 segundos. Na segunda fase, alcançamos 0,088\% de brecha de dualidade em 221,41 segundos, quando paramos a execução. Nesta fase utilizamos como critério de parada a tolerância de $0,1 \%$ de brecha de dualidade. Em nossos testes, com esse critério alcançamos otimalidade. Apesar de utilizarmos somente os dados de um departamento, acreditamos que é possível expandir o uso da ferramenta para todos os departamentos da UFES, campus São Mateus. Por ser uma proposta específica para a UFES, não comparamos nosso modelo com outros da literatura.

Outro trabalho na mesma linha é o desenvolvido por Araujo e Secchin [1]. Nele os autores dão enfoque à eficiência computacional na resolução. O preço é uma excessiva simplificação da realidade: todas as disciplinas têm mesma carga horária. Em nosso trabalho, o modelo é mais representativo da realidade, a condensação é mais eficaz, e o custo computacional aumenta muito pouco. Isso deve-se à estratégia de resolução em duas fases, diferente do trabalho de Araujo e Secchin, onde no mesmo modelo são divididas disciplinas e horários.

\section{Conclusões}

Os resultados obtidos mostram que a modelagem adequou-se muito bem à realidade do Departamento. Optamos por não levar em consideração professores substitutos. Esses docentes têm um regime diferenciado na divisão de disciplinas, e portanto pequenos ajustes devem ser feitos para contemplar essa realidade. Um dos pontos não completamente resolvidos é a divisão das salas. Uma proposta é criar uma terceira fase que aloque as disciplinas nas salas, de modo a evitar que uma mesma turma principal tenha aulas em diversas salas, por exemplo. Notemos que a quantidade máxima de salas é levada em consideração na restrição (1). Isso permite, em tese, ser possível tal estratégia.

\section{Referências}

[1] R.L. de Araujo; L.D. Secchin, O problema da elaboração de grade de horários: estudo de caso em uma Universidade Brasileira, Anais do CMAC Nordeste, 2012.

[2] S. Daskalak; T. Birbas, Efficient solutions for a university timetabling problem through integer programming, European Journal of Operational Research, 160 (2005) 106-120.

[3] S.A. MirHassani, A computational approach to enhancing course timetabling with integer programming, Applied Mathematics and Computation, 175 (2006) 814-822. 